
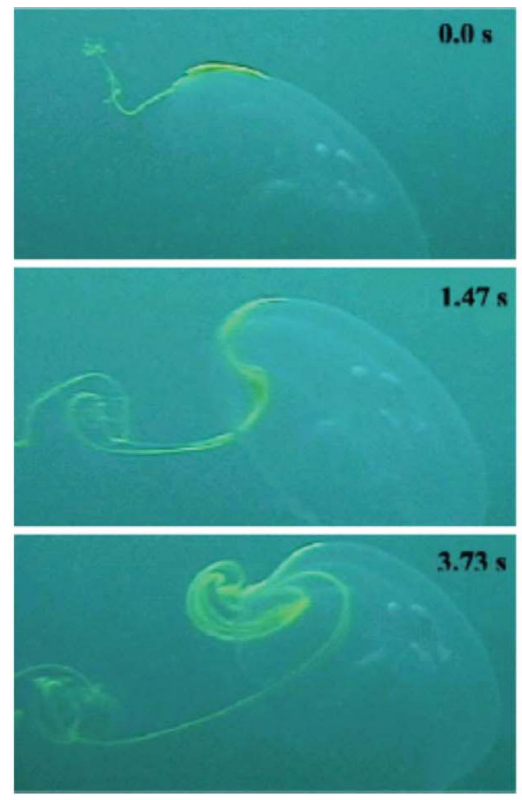

(a)

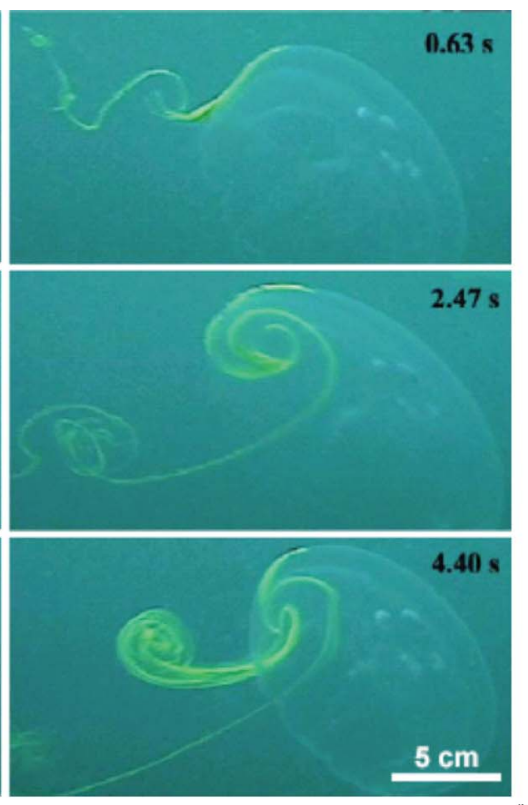

$5 \mathrm{~cm}$

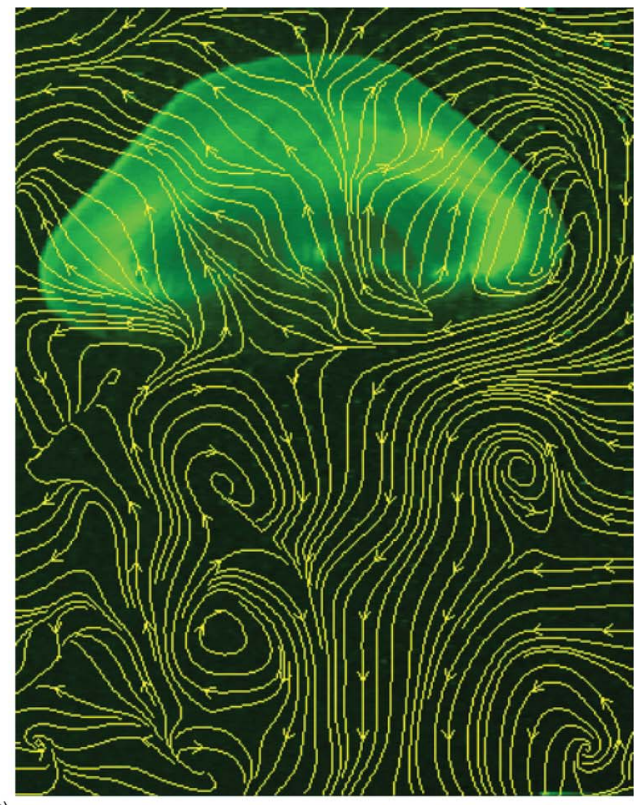

FIG. 1. (Enhanced online). (a) Sequence of two swimming cycles visualized using dye. (b) Instantaneous streamlines of flow measured using DPIV.

\section{Vortex motion in the ocean: In situ visualization of jellyfish swimming and feeding flows}

\author{
John O. Dabiri and Morteza Gharib \\ California Institute of Technology, Pasadena, California 91125 \\ Sean P. Colin \\ Roger Williams University, Bristol, Rhode Island 02809 \\ John H. Costello \\ Providence College, Providence, Rhode Island 02918
}

(Received 24 February 2005; published online 26 August 2005)

[DOI: 10.1063/1.1942521]

We present a combination of both qualitative flow visualizations of jellyfish in their natural marine environment and quantitative measurements in a laboratory facility, to understand how the animals effectively use principles of fluid mechanics in their swimming and feeding behaviors.

Aurelia aurita jellyfish were observed in a natural habitat off the coast of Mljet, Croatia in a marine lake. Video was collected by a scuba diver using natural light. A second diver injected $20 \mu \mathrm{l}$ pulses of concentrated fluorescene dye into the water surrounding the animals. As shown in the images [Fig. 1(a)], the dye was injected upstream of the animal to observe the fluid motion induced by the animal as it swims forward. The formation of a vortex ring is observed as the animal contracts its body. A second vortex ring of opposite rotational sense forms as the animal relaxes to its original resting form. Subsequently, the two vortex rings pair together and develop azimuthal instabilities as they propagate downstream.
Quantitative measurements of similar flows observed in a laboratory jellyfish tank further elucidated the nature of these vortex dynamics. Using digital particle image velocimetry, we have mapped the topology of the vortex wake behind the animals. As shown in Fig. 1(b), a strong entrainment flow is induced near the bell margin by the generated wake vortex rings. This induced flow captures prey and transports it to the feeding surfaces inside the jellyfish. A net momentum flux opposite the swimming direction is observed in the far wake, indicating the generation of unsteady swimming forces.

Although the formation of a vortex ring during body contraction has been previously observed, this work presents the first evidence of formation of an equally prominent vortex ring during body relaxation. In traditional models of pulsatile swimming, the relaxation phase of the swimming cycle is either neglected altogether or accounted by simple quasisteady assumptions. However, given the present observations, it is evident that the vortex dynamics associated with both contraction and relaxation must be included to achieve accurate estimates of swimming dynamics. ${ }^{1}$

We gratefully acknowledge funding from the National Science Foundation (Grants No. OCE-0116236 to J.H.C. and No. CTS-0309671 to M.G.) and Aurelia medusae provided by the Cabrillo Marine Aquarium.

${ }^{1}$ J. O. Dabiri, S. P. Colin, J. H. Costello, and M. Gharib, "Flow patterns generated by oblate medusan jellyfish: Field measurements and laboratory analyses,” J. Exp. Biol. 208, 1257 (2005). 